\title{
L'énonciation du journal mural : une hybridité débridée
}

\section{Annick Monseigne}

\section{(2) OpenEdition}

1 Journals

Édition électronique

URL : http://journals.openedition.org/communicationorganisation/4753

DOI : 10.4000/communicationorganisation.4753

ISBN : 979-10-300-0155-6

ISSN : 1775-3546

\section{Éditeur}

Presses universitaires de Bordeaux

\section{Édition imprimée}

Date de publication : 1 décembre 2014

Pagination : 113-128

ISBN : 978-2-86781-905-6

ISSN : $1168-5549$

\section{Référence électronique}

Annick Monseigne, "L'énonciation du journal mural : une hybridité débridée », Communication et organisation [En ligne], 46 | 2014, mis en ligne le 01 décembre 2017, consulté le 26 avril 2019. URL : http://journals.openedition.org/communicationorganisation/4753; DOI : 10.4000/ communicationorganisation. 4753 


\title{
L'énonciation du journal mural : une hybridité débridée
}

\author{
Annick Monseigne ${ }^{1}$
}

C'est dans l'entrelacs du formel et de l'émotionnel, du dispositif technique et de l'espace cognitif que se nouent des relations étroites entre projet et design. De cette fonctionnalité du projet et de la présence esthétique de l'objet émergent des qualités relationnelles insoupçonnées et innovantes. La communication publique l'a compris en y puisant un nouveau souffle s'inspirant de la culture du partage et de la co-construction du sens.

Cette contribution s'intéresse à la production et la circulation du « sens » et des « signes ", dans un cadre méthodologique qui relève de la communication des organisations publiques. Elle propose d'aborder le concept original du journal mural à partir de l'étude d'un projet éditorial réalisé dans le cadre d'un cours $^{2}$ de stratégie éditoriale en Master Communication Publique et Politique (Isic-IEP) à l'Université Bordeaux Montaigne. La problématique concernait la visibilité et l'accessibilité de l'action culturelle du Rocher de Palmer ${ }^{3}$ (salle de spectacle de la ville de Cenon en Gironde) dans l'espace public cenonnais.

Car comment aborder la question des politiques culturelles sans se questionner sur les liens entre les ouvres et les publics, entre les salles de spectacle et les populations de leurs territoires, entre une offre et une demande ? Force est de constater que tous les citoyens ne se retrouvent pas dans les programmations et qu'une frange de la population fréquente peu ou

\footnotetext{
1 Annick Monseigne est maître de conférences en Sciences de l'Information et de la Communication à l'Université Bordeaux Montaigne et chercheur au MICA - Médiation, information, communication, art - EA 4426. Elle a exercé au sein de sa propre agence comme consultante auprès des collectivités territoriales pendant près de vingt ans ; annick.monseigne@iut.u-bordeaux-montaigne.fr

2 Ce cours de stratégie éditoriale que nous menons en collaboration avec le designer graphiste, David Gimenez, intervenant professionnel à l'Université Bordeaux Montaigne, répondait avec la promotion 2012 du Master CPP (direction : G. Guilhaume) à une commande de la ville de Cenon autour de la problématique : comment rendre visible et accessible l'action culturelle du Rocher de Palmer (salle de spectacle) directement dans l'espace public cenonnais?

3 Le Rocher de Palmer inauguré en 2010 est un complexe de salles de spectacle de $6700 \mathrm{~m}^{2}$ composé de trois espaces modulables : deux salles de spectacle de 1200 et 650 places et un salon de musique; une galerie les relie. Sy rajoutent un centre de documentation, un atelier de travail et un espace restauration. Culturellement, cette structure s'impose comme le cœur battant des « musiques du monde » dans la région.
} 
pas cette salle de spectacle. Ainsi, comment faire pour inverser la tendance et attirer de nouvelles populations ? Comment relier davantage les publics et les œuvres musicales?

En réponse à cette problématique, un journal mural a été proposé. Réalisé par la ville de Cenon en 2013, nous l'analyserons, en tant que projet éditorial, dans sa dimension stratégique, technique et culturelle. Soit un journal mural composé de huit pages de photos, d'illustrations, de bruits et de paroles à lire ou à entendre dans divers espaces publics de la ville. Ces pages monumentales de ce journal mural racontent, haut et fort, des expériences peu ordinaires vécues par des Cenonnais au sein du Rocher de Palmer.

Concernant le design pensé comme élément constitutif du projet éditorial, nous questionnerons, dans une approche sensible, les effets de sens du matériau, de la forme du support et du rapport au corps. C'est l'objet à voir, à toucher, à sentir, à ressentir, l'objet qui détourne l'attention de soi au privilège de l'autre et du collectif qui nous intéresse. La pertinence sémiotique de la pratique, ici déviée de ses fonctions classiques, devient fondamentale pour approcher le journal mural comme objet de design promoteur d'actions et catalyseur d'interactions.

Notre problématique cherche à comprendre en quoi la nature de ce projet éditorial structure les processus d'action du sujet utilisateur et comment le design accélère la performance cognitive du sujet du faire.

Notre travail part de l'hypothèse de la factitivité de l'objet sémiotique, c'est-à-dire le rapport entre la fonctionnalité opératoire et la fonctionnalité communicative de l'objet (Deni, 2005 : 83). Nous chercherons donc à analyser le fonctionnement factitif du journal mural, autrement dit sa capacité à « faire-être » le monde, installer le citoyen au cœur de l'objet et l'entraîner dans le "faire-faire " de la pratique quotidienne. Notre postulat de base suggère la réversibilité du faire : on peut faire avec et à travers l'objet mais il peut aussi nous faire faire quelque chose. Factitif par intention, le journal mural résultant pour partie d'un processus de design est abordé en termes sémiotiques de forme/action.

Une première partie descriptive sera consacrée à la contextualisation et la précision de notre cadre épistémologique. Une seconde partie analytique permettra d'interpréter dans une approche de sémiotique-objet les relations dialogiques entre projet et design juste avant d'esquisser l'organon qui décrira le principe de journal mural en tant qu' « objet factitif » (Deni, 2005).

\section{Contexte et détour historique}

Pourquoi certains habitants hésitent-ils encore à pousser les portes du Rocher de Palmer, malgré des tarifs serrés et des places gratuites ? Dès 2011, toute l'équipe de la direction de la communication ${ }^{4}$ s'est saisie de la question.

4 Ce service communication dirigé par Marie-Hélène Filleau est composé de quatre secteurs (publication print et web, communication interne, communication externe et production, impression). 
En 2012, vingt-cinq étudiants de Master 2 du parcours CPP se sont joints à la réflexion et ont travaillé sur un projet de journal mural de proximité. Une idée suggérée par David Gimenez de l'agence bordelaise de communication visuelle E $3^{5}$. Pour ce designer graphique, ce principe se situe « entre le Street Art (un style de journal mural revendicatif) et une esthétique qui peut, $a$ priori, sembler un peu décousue. Le journal doit étonner d'emblée, permettre l'accès à une information claire, en rupture totale avec celle très codifiée de l'information publicitaire qui sature le milieu urbain $»^{6}$. C'est ce qu'a fait la ville de Cenon et son service communication. Un détour par l'histoire de l'évolution du journal mural s'impose avant de disséquer la nature et le contenu du projet éditorial qui nous occupe.

\section{L'histoire du journal mural ${ }^{7}$}

Sous la Révolution française, la presse connaît une expansion sans précédent, passant de quelques publications à environ 1350 journaux. Bon nombre des journaux de la période sont conçus comme des affiches, lesquelles sont ensuite placardées dans les rues, lues et commentées sur la place publique. Nous retrouvons le même enthousiasme et le même développement sous la Commune de Paris. Cet enjeu de l'accès à l'information et à la prise de position politique caractérise la diffusion des supports partout dans le monde. Ainsi, l'expression de l'opinion publique par l'affichage est une tradition de la Chine impériale.

\section{Un outil de contestation}

Ces « affiches de grand caractère " (dazibao) sont placardées dans la ville et jusque dans la rue devant le tribunal, siège du magistrat. Le peuple se rassemble autour des affiches pour les commenter. En 1966, lors de la Révolution culturelle lancée par Mao Zedong, les dazibao refont leur apparition. Très répandus, ils sont utilisés aussi bien pour débattre, qu'à des fins satiriques ou de dénonciation. Réalisées à la main, ces dazibao couvrent d'abord les murs de Pékin avant de gagner les provinces. Ce média illégal et spontané véhicule l'information non officielle et a l'audace d'attaquer les autorités du pays.

De l'outil d'expression individuelle à l'espace de création dynamique

Mai 2012, dans le $\mathrm{IX}^{\mathrm{e}}$ arrondissement de Paris, un animateur radio sans emploi, est sur le point d'être expulsé de son logement. Depuis trois semaines, il tient un journal muraß sur la façade de son immeuble, afin de raconter son

\footnotetext{
5 Disponible sur http://www.e3agence.fr/

6 Disponible sur http://www.ville-cenon.fr/culture-loisirs/rocher-de-palmer/1287-la-clameur.html. Consulté le 3 avril 2014.

7 Disponible sur http://www.lecridupeuple.org/de-laffiche-murale-au-blog-permanence-du-media-populairepolitique/. Consulté le 4 avril 2014.

8 Disponible sur http://www.lesinrocks.com/2012/06/12/actualite/un-journal-mural-pour-raconter-son-
} 
histoire. Les feuilles s'accumulent, sagement scotchées ou clouées les unes à côté des autres. La nuit suivante, il placarde des affiches sur sa porte et ses fenêtres. Chaque jour, il en ajoute de nouvelles, se livrant un peu plus à chaque fois allant même jusqu'à suspendre des ampoules qui symbolisent la coupure d'électricité.

Face aux mutations des questions et problématiques sociales, la dynamique induite par le journal mural devient un espace de création pour une mise en scène et en mots de pratiques sociales émergeantes. Contrairement à JeanPierre Boutinet qui voit le projet comme un symptôme de l'individualisation en le réduisant à un «bricolage de petits idéaux » (Boutinet, $1990: 2$ ), ici, il est envisagé dans sa dimension " projective » comme moyen d'expression et d'ouverture à d'autres possibles.

\section{Deux projets en un ?}

En réalité avec la notion de projet éditorial nous sommes en présence de deux questions de recherche, l'une corrélée aux techniques de projet, l'autre à l'organisation par projet.

\section{Les conduites à projet}

De l'approche anthropologique du projet de Boutinet nous retenons, dans ce qu'il nomme les organisations à projet, " le projet expérimental et participatif » (1990: 116). La gestion par projet est alors une manière de penser l'alternative au fonctionnement bureaucratique des organisations en développant le capital de motivation et de créativité du potentiel humain. En entendant stimuler un haut niveau de créativité et de performance ainsi qu'une forte implication des membres concernés, ce projet de journal mural en tant que nouvel outil de communication éditoriale s'avérait d'une grande richesse au plan communicationnel. Pour autant, nous n'aborderons pas ce versant organisationnel dans le cadre de cette étude, préférant nous consacrer aux techniques encore très présentes dans le domaine du projet, et plus précisément du projet dit «d'opportunité culturelle » $(1990: 298)$ pour reprendre la terminologie de Boutinet.

\section{Les techniques de projet}

Techniquement, si nous devions définir de manière classique le projet éditorial, nous évoquerions inévitablement la ligne éditoriale qui définit les priorités, les champs d'intérêt du contenu rédactionnel du journal et qui contribue à la cohérence et à la crédibilité de ce dernier. Cinq éléments principaux définissent la ligne éditoriale : l'audience cible, le produit, la concurrence, le contenu éditorial et la tendance politique du journal. S'agissant $\mathrm{du}$ contenu éditorial et du somatexte ${ }^{9}$ qui l'accompagne, c'est-à-dire la

expulsion-11268211/. Consulté le 5 avril 2014.

9 Le somatexte est le lieu d'expression de l'identité d'un journal, une sorte de mise en scène de l'imprimé qui 
définition du climat de lecture, c'est à cet endroit précis du projet éditorial que la nécessité d'une démarche heuristique s'est faite ? sentir. Pour rompre l'image du Rocher de Palmer apparu comme une structure monumentale et inaccessible ${ }^{10}$, les étudiants du parcours $\mathrm{CPP}$ ont proposé d'engager une démarche expérientielle en positionnant ce nouvel outil d'information et de communication hors des standards habituels de la publication et de l'édition. Un support décomplexé, simplifié, ludique et accessible est alors pensé pour casser la barrière érigée entre le Rocher de Palmer et la population cenonnaise ${ }^{11}$. L'aspect ludique passant d'abord par la forme, le journal envisagé alors comme un objet décide de sortir de l'espace intime (prise en main individuelle) pour investir l'espace public (forme de lecture partagée) en assumant sa présence physique dans le paysage urbain. Le choix du concept de journal mural permettait ainsi d'offrir des modalités de réception plus riches que celles d'un journal papier classique puisqu'en plus de solliciter la vue, ce sont les cinq sens du récepteur qui sont mobilisés. Bien entendu, cela suppose de ne pas tomber dans le piège de reprise des spécificités de l'affichage ou du magazine, l'intérêt étant de s'adapter d'abord aux environnements de pratiques du média, puis aux fonctionnalités propres de l'outil pour faire émerger une forme de pragmatique éditoriale (Piponnier, 2006).

\section{De l'objet au design}

Dans notre quête du design que nous avons sentie d'emblée en balance entre la technique et l'art, le produit et l'œuvre, nous décidons de nous aventurer dans un discours sur l'objet pris au sens large du terme, c'est-à-dire en tant qu'objet culturel et outil libérateur de la servitude. Un détour par la sémantique nous apprend que le mot anglais renvoie à un double sens qui conforte le lien pressenti au graphisme (dessin) et au projet (dessein), soit la projection consciente par un acte d'inscription matérielle d'un objectif à atteindre.

\section{Design et technique}

Le design c'est aussi le mariage conflictuel de l'art et de la technique, de l'esthétique et de l'utilitaire. Nous retenons quelques éléments définitionnels dans le champ catégoriel de base. La technique, du grec techné se définit

en appelle à l'émotionnel. Il comprend : le choix du support, du papier, de la typographie et la définition du champ chromatique. Voir Charles Bonnet, « La communication imprimée », Communication et langages, $\mathrm{n}^{\circ}$ 86, 1990, p. 37-52.

10 Selon une pré-enquête menée par les étudiants CPP et dont les conclusions ont montré : une connaissance et une bonne appréciation du lieu par les habitants (hors quartiers excentrés), une programmation mal connue et peu adaptée (surtout auprès des jeunes et des personnes âgées), une problématique des tarifs peu impactante, le manque de temps comme obstacle.

11 En termes de logement et de catégories socio-professionnelles on constate que le niveau de vie de la population cenonnaise est bien inférieur à la moyenne nationale. Cenon qui a également pour caractéristique d'avoir une population multi-ethnique, peu diplômée, est également une ville classée en Zone d'Éducation Prioritaire. 
avant tout comme un savoir-faire. Qu'il s'agisse de procédés pour arriver à une fin ou de procédés de fabrication, dans tous les cas l'outil, l'utilitaire et la valeur marchande ne sont jamais bien loin. Mais parce que la techné est comme le disait Aristote (1965: 173) « une disposition accompagnée de raison et tournée vers la création ", englobant art et technique, elle est aussi le prolongement de son intelligence et de sa forte capacité créative. Il nous reste maintenant à nous repérer dans le champ très vaste « des designs » (Quinton, 1998). Parmi les catégories existantes, celle dite de « design graphique » en tant que processus de communication qui utilise les signes graphiques, objets et espaces nous semble parfaitement adaptée aux caractéristiques de notre objet considéré comme lieu privilégié de la médiation du champ social par la création. Utilisons maintenant la sémiotique pour mieux comprendre ce concept de journal mural et son activité de sémiotique-objet présentée comme un dispositif signifiant au fort pouvoir interactionnel et relationnel.

\section{Sémiotique et design}

Pour nous situer, nous nous appuyons sur le travail de théoriciens pour tracer quelques axes structurants nous aidant à la construction de notre analyse. Les objets au quotidien (Fontanille et Zinna, 2005), les pratiques sémiotiques (Fontanille, 2008) et la sémiotique du design (Beyaert-Geslin, 2012), nous servent de repères tout au long de ce travail.

Les typologies de signes étant de plus en plus complexes, nous préférons nous intéresser à ce que les images font, et aux actes qu'elles accomplissent, pour nous, au quotidien. Parce que les images ont le pouvoir de modification des éléments de la réalité, parce qu'elles «font être » des éléments de la réalité et nous « font faire » quelque chose dans la réalité, c'est dans ce que Fontanille nomme les pratiques sémiotiques que nous nous inscrivons. Sorte d'escapade hors du texte, la sémiotique-objet s'intéresse notamment au design d'objets. Le journal mural en tant qu'hybridation de stratégies éditoriales (message graphique et rédactionnel) et de principe d'affichage urbain (support et public) sera éprouvé au plan de l'immanence des pratiques notamment à travers le rapport qui s'établit entre l'environnement, les objets, les stratégies d'acteurs mais également les corps (sujet et objet).

\section{Médiation sémiotique et énonciation éditoriale}

Pour Emmanuel Souchier (1998, 2007) spécialiste de l'énonciation éditoriale, la consécration de l'écriture n'équivaut pas à l'actualisation de la lecture. Or, c'est bien dans ce que Yves Jeanneret $(1998,2008)$ a nommé " espace de trivialité » que circulent les idées, les images, les objets culturels et que la dynamique de l'interprétation opère. C'est dans ce complexe intersémiotique, et dans le cas du journal mural, dans la matérialité du support et son organisation graphique, typographique, photographique que se jouent les enjeux de la médiation, par et pour les hommes. 


\section{À la recherche de nouvelles formes sémiotiques}

Cette médiation sémiotique se veut singulière parce que double avec une dimension organisée et une ouverture possible à l'interprétation et donc à une mobilisation active du destinataire (récepteur). Nous considérons, dès lors, que ce qui est envisagé, c'est que les rapports de la population cenonnaise avec le Rocher de Palmer passent désormais par une certaine forme d'écriture et de condition d'interprétation. Le journal mural en tant qu'outil de communication destiné à l'interprétation est saisi par des cultures, des normes et des codes très éloignés des formes sémiotiques organisées, classiques et reconnues au sein de notre société. À la croisée de la technique et de la poétique, ces nouvelles formes sémiotiques ont pour mission de transformer les comportements des Cenonnais et au-delà de changer leurs conditions d'Homme. C'est par le biais d'un engagement de création quasi poétique de la part des éditorialistes et techniciens du projet que le miracle doit avoir lieu.

\section{Les pouvoirs de "l'image du texte"}

Les traces sémiotiques laissées par chaque corps de métier (rédacteur, graphiste, maquettiste, imprimeur) révèlent dans leurs dimensions visuelles leur pluralité énonciative. Et c'est à cette « énonciation sur la page » (Souchier, 2007 : 27), c'est-à-dire ce que l'on pourrait appeler le non verbal de l'édition qui " ne peut se dire avec des mots ", que nous faisons référence. À une époque, qui plus est, où les pratiques et les modes d'écritures sont en pleine mutation, y compris dans le champ de la communication publique (Monseigne et Guilhaume, 2012), changer de mode d'expression, vouloir surprendre le lecteur en le confrontant à une praxis encore peu absorbée par l'épaisseur de l'usage et de l'usure nous semble pertinent. Ainsi, "l'image du texte » pour reprendre Souchier est une part constitutive de l'énonciation éditoriale. Pour autant toute écriture relève de la linguistique et de l'iconique, c'est donc à ces deux niveaux d'expression augmentés d'une instance matérielle, c'est-àdire le support formel, que nous ferons appel pour décrire notre organon, soit un ensemble de règles pratiques appliquées ici à notre objet issu de la sémiotique dynamique et du sensible. C'est le pouvoir de communicabilité du journal mural que nous allons analyser à travers la décomposition de cette forme d'organisation sémiotique.

\section{Une esquisse d'organon}

Après avoir travaillé séparément les notions de projet et de design, nous allons les penser ensemble par le biais du fonctionnement factitif de notre objet. C'est sur l'expérience non plus de conception (2012) mais de réalisation du produit (2013) qui s'est faite hors circuit universitaire entre le designer graphiste et le commanditaire, que nous avons choisi de nous appuyer pour bâtir l'organon sémiotique du journal mural en tant qu'objet technique. 
La pluralité des voix (métiers) qui participent à cet orchestre nous intéresse au premier chef pour décrire l'énonciation éditoriale du journal mural dans sa relation de la forme au sens.

\section{Un déplacement de l'affiche vers le journal mural}

Ce n'est pas qu'un type d'objet (l'affiche) qui fait l'objet-support (journal d'information), l'acte de lire le journal sur un support mis en scène dans l'espace public (sur un panneau, un mur, au sol) grâce au travail iconographique et textuel (designer graphiste et communicant) peut aussi faire l'objet-support. Et ce sont les différents types de supports, dont certains détournés (une affiche en journal mural), qui contribuent à un niveau de pertinence sémiotique. L'objet support (un affichage décalé) est une sorte d'interface qui définit une relation déictique (c'est ici) et une relation modale (attirer l'œil) pour «faire faire ", et en l'occurrence, faire lire (factitif).

\section{Le cadre comme puissant processus d'induction}

Si l'on examine ce journal, on constate que ces huit pages de 1 mètre de haut et $75 \mathrm{~cm}$ de large seront apposées sur des supports non classiques, voire non attendus, c'est-à-dire des supports (panneau, mur, sol) capables de rompre avec un cadre purement institutionnel (panneaux de mobilier urbain de type affichage publicitaire ou de campagne électorale) ou marketing. Il s'agit de mettre en scène dans la ville et dans une dimension stratégique un principe d'affichage, en apparence sauvage, susceptible de garantir l'équilibre entre lisibilité et visibilité (Amri et al., 2007).

Ici la population cenonnaise se trouve prise dans des processus d'induction repérables dans différents domaines de la communication (Hotier, 1997), les uns guidés par le cadre (approche par la scène ou mise en scène), mais aussi par le sens (approche par les stratégies d'influence) ou le sujet (approche par la sensorialité, la sensualité).

Des partis pris graphique et d'impression unis dans la force réceptive
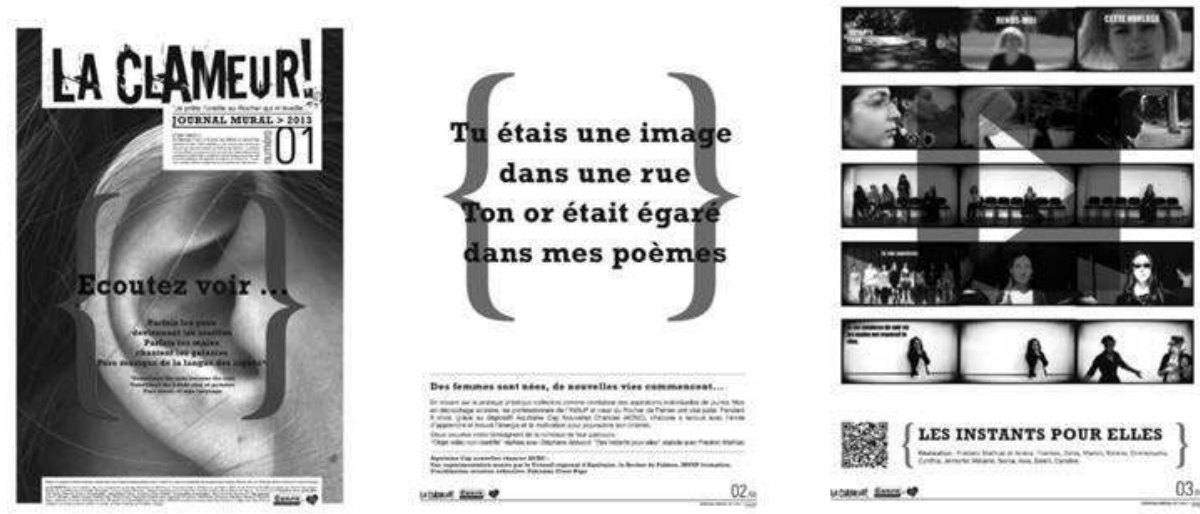

Figure 1 : Poème de Kamel Daoud - Figure 2 : Les instants pour elles (1) - Figure 3 : Les instants pour elles (2) Graphisme : David Gimenez/agence E3 -

Visuels : Service communication de la mairie de Cenon, Jean-Paul Nogues 

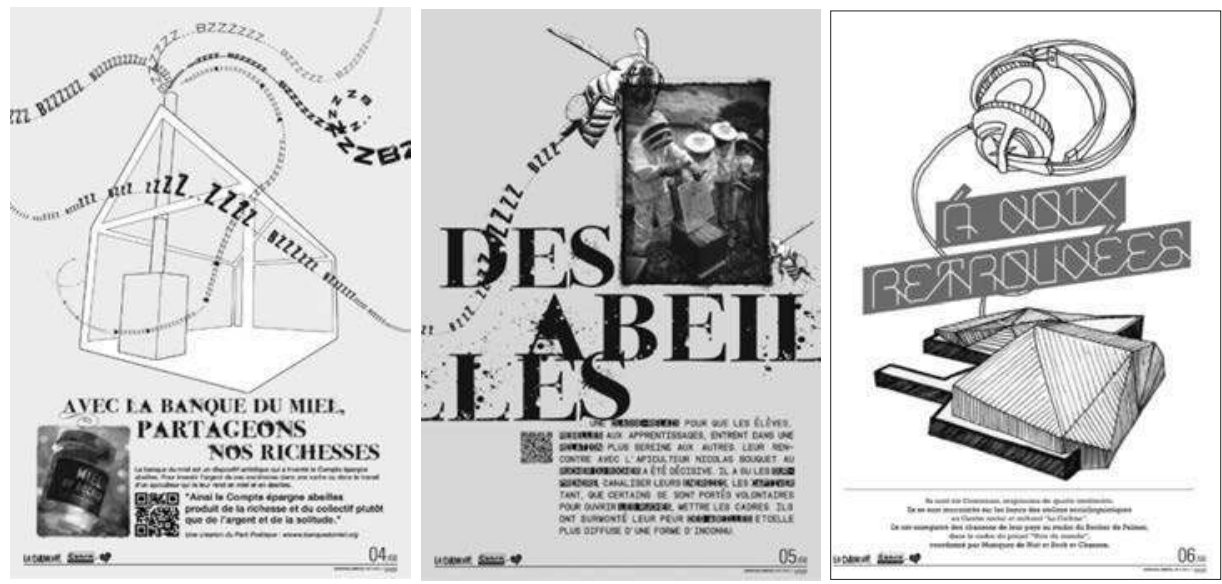

Figure 4 : Le Rucher du Rocher (1) - Figure 5 : Le Rucher du Rocher (2) - Figure 6 : À voix retrouvées (1)

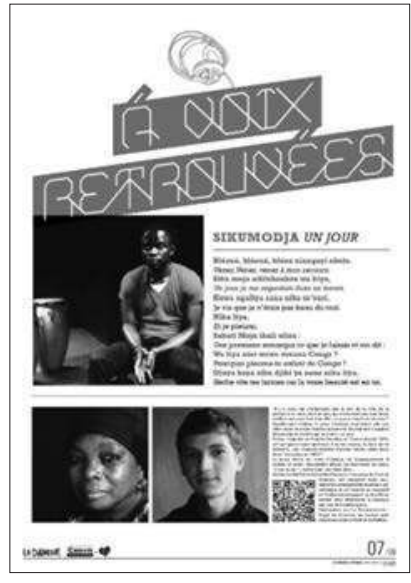

Figure 7 : A voix retrouvées (2)

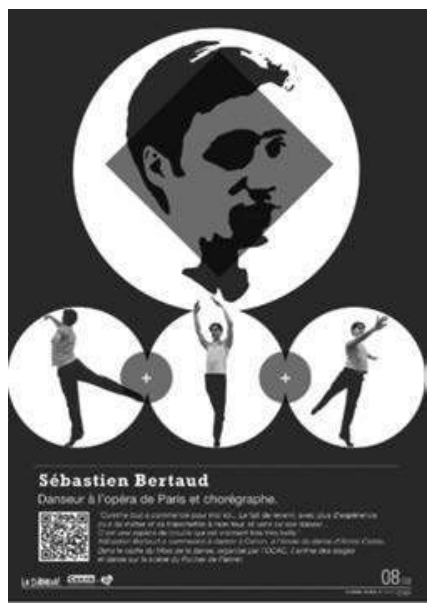

Figure 8 : Chorégraphie de Sébastien Bertaud

Graphisme : David Gimenez/agence E3 -

Visuels : Service communication de la mairie de Cenon, Jean-Paul Nogues

L'impression sérigraphique artisanale à "l'insoleuse » est un des partispris artistiques retenus. La sérigraphie, bien connue des illustrateurs de bande dessinée, est une aventure d'artisanat d'art qui s'apprivoise, se dompte durant les différentes étapes de fabrication. Une technique de pochoir permet de travailler la décomposition de l'image. Le rendu visuel de ce travail de sérigraphie est exceptionnel en terme de force réceptive car l'œil est attiré par l'intensité des couleurs. Pour finir, l'impression sérigraphique est la garantie d'une couleur qui dure dans le temps, et d'une palette chromatique originale (fluo, argentique, métallique). 
Sur le plan graphique les tons sont chauds et saturés, les polices de caractère et les images se manifestent avec excès au plan figuratif. L'humain est à l'honneur et la nature convoquée. Le style est épuré et l'approche se veut définitivement " ontologique ", c'est-à-dire au plus profond de l'Être et au plus près de son expérience. Ainsi, des éléments graphiques qui se veulent en adéquation avec des signes d'authenticité reviennent régulièrement et tous subsument la nature. Des organes humains sont zoomés, des corps s'offrent en mouvement, des insectes sont caricaturés, des sons convertis phonétiquement. Cependant, la construction de la scène doit être complétée par d'autres propriétés que cette mise en forme spécifique.

\section{Texte-énoncé et performativité}

Les accroches verbales de ces huit pages de journal mural portent des injonctions directes : "écoutez voir... ", «(...) partageons nos richesses ", « rends-moi ». Elles interpellent et impliquent le spectateur en tant qu'acteur individuel avec l'utilisation de marqueurs discursifs (tu, nous, vous). En outre, les interactions texte-sujet sont toujours de nature exclusive puisque les Cenonnais (usagers) découvrent les pages du journal mural les unes après les autres.

$\mathrm{Au}$-delà des actes de langage, la performativité se lit aussi dans une forme de lyrisme et de poésie récurrente dans le discours. Sur la première page une oreille gigantesque donne le ton de l'approche ontologique avec cette accroche « écoutez voir » qui joue sur la complémentarité des cinq sens. C'est comme si un voyage intérieur accompagné de quelques promesses poétiques était annoncé. En réalité, c'est la factitivité qui commence déjà à opérer. L'objet en tant qu'instance nous affronte (Beyaert-Geslin, 2012), les corps (objet et sujet) en présence dialoguent, les forces s'ajustent mutuellement. Sur la deuxième page du journal mural un extrait d'un poème réalisé par un groupe de jeunes filles en décrochage scolaire est porteur de passion : " tu étais une image dans une rue, ton or était égaré dans mes poèmes ». Encadrées notamment par des professionnels du Rocher de Palmer, ces jeunes filles se sont essayées à la pratique artistique collective avec succès. La dernière page met en scène un danseur de l'opéra de Paris, autrefois élève de l'école de danse de Cenon. Aujourd'hui chorégraphe et animateur de stages au Rocher de Palmer, il exprime sa satisfaction à « (...) revenir avec plus d'expérience (...) et transmettre à son tour (... )». Et puis, il y a le rucher du Rocher et cette classe-relais (collégiens en rupture scolaire) en quête d'aventure collective et d'apprentissage relationnel. Au fond, on peut lire ici comme sur l'ensemble des pages du journal mural cette volonté, en filigrane, de valoriser le Rocher et la ville de Cenon par le biais d'expériences singulières mais toujours exemplaires des habitants. Par cette double aspectualisation à la fois singulative (événements exceptionnels) et itérative (pratiques du quotidien), ces actes de langage participent au pouvoir de captation de notre sémiotique-objet. 


\section{Les actes spécifiques du journal mural ${ }^{12}$}

Ainsi nous avons repéré un acte :

- d'ostentation et d'intensification qui se manifeste par la taille du journal qui adopte le format de l'affiche, mais aussi la taille des éléments graphiques (image, typographie, etc.) grossis à l'excès ou la surprésence des images et des illustrations (60\% d'images pour $40 \%$ de textes) ;

- d'identification grâce à une compétence sémantique, autrement dit la thématisation du medium (la première page avec sa mention "journal mural ", son titre " La clameur » et le $\mathrm{QR}$ code du site de la ville), et à une compétence modale capable de présenter un fait comme possible, ici non pas avec les marqueurs habituels (pouvoir, vouloir, etc.) mais implicitement par le biais d'une mise en scène forte. Exemple : un danseur de l'opéra décide de revenir danser dans la commune qui l'a vu grandir. Ces motifs modaux et pathémiques participent d'une sorte de connivence sociale ;

- de sélection dans la mesure où le cadre décalé induisant une mise en scène identifiée hors des circuits urbains traditionnels est captatif et où les données textuelles triées et réduites se donnent à consommer comme une denrée rare.

\section{La factitivité intentionnelle de l'objet}

La transformation d'un journal d'information traditionnel (format publication classique), induisant une prise en main de l'objet, en journal mural (format affiche), impose le corps en action de l'usager. Ces transformations prédisposent à un mode de lecture et de comportement spécifiques. Ce sont maintenant aux éléments fortement factitifs (format, niveaux de lecture, couleurs, typographie) qui guident l'action des Cenonnais pour accéder, dans un premier temps, au contenu d'information (message), dans un second temps, à l'outil culturel (le Rocher de Palmer), que nous souhaitons nous intéresser.

- Le format inhabituel pour un journal $(1 \mathrm{~m}$ par $75 \mathrm{~cm}$ ) impose une lecture debout et induit un déplacement physique. La lecture peut s'organiser soit en séquences individuelles, soit globalement. Dans ce dernier cas, si les huit pages sont disposées successivement, le déplacement physique se fait latéralement, tandis que dans l'optique d'une configuration sphérique (agora) de l'agencement des pages, la lecture est circulaire et se voit augmentée d'une fonction d'échanges et de débat dans l'espace public.

- Quatre niveaux de lecture en moyenne (titre, chapeau, texte, image) caractérisent le journal mural, autrement dit, plus que l'affiche et moins que le journal traditionnel. Or, compte tenu du format monumental, lui-même

12 Nous avons pris appui sur la méthodologie de l'« étude de cas : l'affichage " de Jacques Fontanille, in Pratiques sémiotiques, Paris, PUF, 2008, p. 179-215. 
entraînant une exagération des distances de pratique de lecture (autour de 5 à 6 mètres au plus loin), un jeu de va et vient physique peut s'installer en fonction de ces différents niveaux de lecture. Une occasion supplémentaire de rendre le lecteur actif dans sa recherche de contenu et d'augmenter sa capacité de «vouloir lire». Graduer la stratégie d'affichage dans l'affichage constitue une piste à explorer.

- La couleur est réduite à un choix de deux ou trois coloris en vue d'obtenir des tons francs et de se distinguer d'une communication institutionnelle ou marketing exclusivement quadrichrome. Des couleurs saturées et intenses qui accrochent l'cil, mais également des typographies de création au style presque chaotique, proche du graphe ou du tag, des polices de caractère à forte densité pour les titres ou les onomatopées. Cette spontanéité recherchée est en parfaite cohérence avec la volonté d'interpeller le lecteur.

D’un point de vue sémiotique, les marques énonciatives présentes dans l'objet journal mural sont nombreuses. Les transformations de comportement de l'usager adviennent pendant la pratique de lecture. Ici, la sémiosis s'exprime à travers la sensorialité du discours et en provoquant, souvent à l'insu de l'usager, l'acte culturel. Du fait que l'action de "faire-faire " passe, non seulement par la perception visuelle et les interactions corps-objet (design d'objet), mais aussi par le propos culturel sous-jacent contenu dans la valorisation de l'individu (projet éditorial), nous pouvons en déduire que le journal mural conjugue efficacement la fonctionnalité communicative (perception) et la fonctionnalité opératoire (usage) avec peut-être un déséquilibre en faveur de la dimension cognitive, autrement dit communicative.

\section{Conclusion}

Après un rappel de la relation du design au projet, nous concluons cette étude sur l'ouverture d'un questionnement communicationnel propre aux organisations publiques et politiques. Nous avons vu que le design pensé et associé en étroite complémentarité avec le projet éditorial, en se situant au-delà de l'improvisation et du "bricolage " (Boutinet, 1990), offre une véritable pratique éditoriale dont les éléments de cohérence ont été repérés. L'énonciation éditoriale en mobilisant la trilogie factitive du journal mural, soit un artefact qui fait être la vie (faire être) de telle manière qu'il conditionne et fait faire au citoyen («faire-faire »), voire fait lire (« faire lire »), l'expérience d'un nouvel environnement culturel, prouve sa valeur et s'impose comme une avancée communicationnelle dans le remodelage volontaire de l'activité éditoriale des organisations publiques.

Aussi, compte tenu du pouvoir factitif du journal mural, de ses velléités provocatrices presque impudiques à montrer ce que d'autres supports de communication effacent, de sa capacité à capter le public en dépoussiérant le propos du politique, pourquoi compte-t-on encore trop rarement des actions de communication inspirées de modèles associant mouvements verticaux 
et horizontaux ? Cet ensemble institutionnalisé de pratiques mettant en relief des normes et une standardisation des produits (contenu et identité graphique) ne gagnerait-il pas à sortir de ces modes de rationalité pour finir par répondre à un changement paradigmatique de la communication publique (Bessières, 2009) ? À l'heure du web collaboratif, des dispositifs participatifs et normes délibératives (Blondiaux et Sintomer, 2002) sommés d’intégrer la population au partage de l'expression démocratique, comment ne pas penser la co-construction d'une communication jusqu'ici normative et de promotion institutionnelle classique ? Ainsi, à une époque de défiance envers la classe politique (Rosanvallon, 2006), le rôle central que joue le citoyen au cœur du mouvement de renouveau démocratique doit servir de cap aux communicants. A fortiori, quand la réalité économique des collectivités territoriales invite à faire appel au «bon sens » du citoyen au profit d'une rationalité économique mais aussi environnementale. Diffuser large tout en ciblant juste - puisque ici un seul numéro du journal peut être vu par l'ensemble de la population -, en pratiquant une communication chirurgicale de précision reste un exemple à retenir. Faire évoluer les comportements compte parmi les missions phares de la communication publique. Loin du marketing territorial et de sa boîte à outil au service de la seule attractivité, cette dernière s'attache avant tout à la démultiplication des formes de médiations et d'expressions entre les citoyens et leurs institutions.

\section{BIBLIOGRAPHIE}

AMRI Mahdi, CANO Anne-Laure, CATOIR Marie-Julie, LECHENAUT Émilie, VERSEL Martine (2007), «Un nouveau regard sur l'affichage urbain : le passage Saint Rémy à Bordeaux », Communication E Organisation, n 32, p. 124-148.

ARISTOTE (1965), Éthique à Nicomaque (IV s. av. J.-C.), Paris, Garnier Flammarion, L. VI, chap. 4.

BEYAERT-GESLIN Anne (2012), Sémiotique du design, Paris, PUF (Formes sémiotiques).

BESSIERES Dominique (2009), « La définition de la communication publique : des enjeux disciplinaires aux changements de paradigmes organisationnels », Communication EOrganisation, $\mathrm{n}^{\circ}$ 35, p. 15-28.

BLONDIAUX Loïc, SINTOMER Yves (2002), "Limpératif délibératif », Politix, Volume 15, n' 57, p. 17-35.

BONNET Charles (1990), "La communication imprimée ", Communication et langages, $\mathrm{n}^{\circ} 86$, p. 37-52.

BOUTINET Jean-Pierre (1990), Anthropologie du projet, Paris, PUF.

DENI Michela (2005), "Les objets factitifs ». In Jacques FONTANILLE et Alessandro ZINNA (dir.), Les objets au quotidien, Limoges, Presses Universitaires de Limoges, p. 79-96. 


\section{$C \& O n^{\circ} 46$}

FONTANILLE Jacques (2007), Affichages : de la sémiotique des objets à la sémiotique des situations, Revista dell'Associazionze di studi Semiotici, disponible sur http://epublications.unilim.fr/revues/as/1113

FONTANILLE Jacques (2008), Pratiques sémiotiques, Paris, PUF.

FONTANILLE Jacques, ZINNA Alessandro, dir. (2005), Les objets au quotidien, Limoges, Presses Universitaires de Limoges.

GALINON-MÉLÉNEC Béatrice, MARTIN-JUCHAT Fabienne, dir. (2007), Le corps communicant - Le XXI siècle, civilisation du corps? Paris, L'Harmattan.

GRAMACCIA Gino, coord. (1998), « Management de Projet et logiques communicationnelles ", Communication E Organisation, $\mathrm{n}^{\circ} 13$, disponible sur http:// communicationorganisation.revues.org/2018.

HOTIER Hugues (1997), "L'induction, paradigme caché de la communication ? ", Communication E'Organisation, $\mathrm{n}^{\circ} 12$, disponible sur : http://communicationorganisation. revues.org/1978

JEANNERET Yves (1998), «L'affaire Sokal: comprendre la trivialité », Communication et Langages, $\mathrm{n}^{\circ} 118$, p. 13-26.

JEANNERET Yves (2008), Penser la trivialité. Volume 1 : La vie triviale des êtres culturels, Paris, Hermès-Lavoisier.

MONSEIGNE Annick, GUILHAUME Geneviève, coord. (2012), « La mutation du métier de Communicant public ", Communication E' Organisation, n 41.

PIPONNIER Anne (2006), « Dispositifs éditoriaux émergents dans les communautés scientifiques internationales de réseau ». In Ghislaine CHARTRON et Évelyne BROUDOUX (dir.), Document numérique et société, Paris, ADBS, p. 251-264.

QUINTON Philippe (1998), «Les designs comme processus de communication », Communication et langages, $n^{\circ} 115$, p. 81-86.

ROSANVALLON Pierre (2006), La contre-démocratie, la politique à l'âge de la défiance, Paris, Seuil.

SOUCHIER Emmanuel (1998), «L'image du texte, pour une théorie de l'énonciation éditoriale ", Les Cabiers de médiologie, n ${ }^{\circ}$ 6, p. 137-145

SOUCHIER Emmanuel (2007), "Formes et pouvoirs de l'énonciation éditoriale ", Communication et Langages, $\mathrm{n}^{\circ} 154$, p. 23-38.

Résumé : Le but de cet article est de penser ensemble les notions de projet et de design pour montrer le pouvoir de communicabilité d'une sémiotique-objet qui s'exprime dans et hors le texte. Le concept de journal mural, forme de médiation du champ social par la création et d'interface des interactions entre les hommes (citoyens) et leurs organisations (publiques), est ici disséqué de manière chirurgicale. Une esquisse d'organon montre le pouvoir de factitivité du journal mural conjuguant efficacement une double fonctionnalité opératoire et communicative.

Mots-clés : projet, design, journal mural, sémiotique-objet, factitivité. 


\begin{abstract}
The objective of this research paper is to "think together" the notions of project and design in order to show the power of communicability of an object-semiotic which finds its expression in and out of the text. The concept of wall newspaper, a form of mediation of the social field through the creation and the interface it builds between people (citizens) and their organisations (public sphere), is dissected in a surgical way. A sketch of organon shows the factivity power of the wall newspaper which combines efficiently a double functionality, both operative and communicative.
\end{abstract}

Keywords: project, design, wall newspaper, object-semiotic, factivity. 
